\title{
An LHCb general-purpose acquisition board for beam and background monitoring at the LHC
}

Topical Workshop on Electronics for Particle Physics Aachen, Germany, 24 September 2010

F. Alessio, R. Jacobsson, CERN, Switzerland

Z. Guzik, IPJ Swierk, Poland 


\section{LHCb Intro: Beam and Background Monitoring @ LHCb \\ ONLINE}

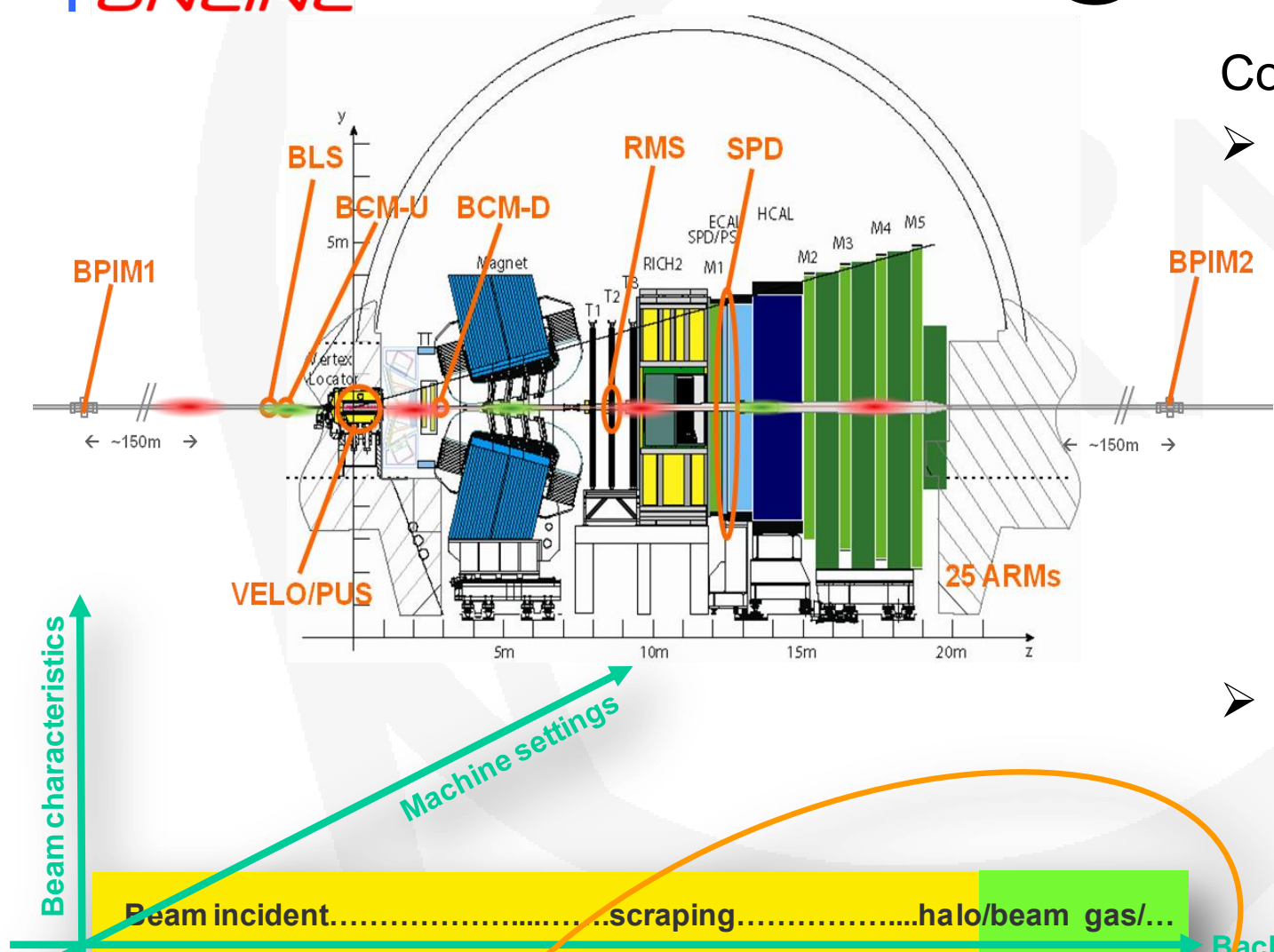

Instantaneous damage.
Trigger rates

Poor data quality

Single event upsets.

Accelerated aging.

Long-term damage

Complete study framework which involves:

$>$ Background monitoring

- Beam Condition Monitor

- Metal-foil based Radiation Monitoring System

- Active Radiation Monitors

- Beam Loss Scintillator

- VELO/PUS

- SPD

$>$ Beam monitoring

- VELO/PUS (and indirectly also the others above)

- Beam Phase and Intensity Monitor ckground 


\section{LHCb BPIM-BPTX system}

ONLINE

It is of extreme importance that the phase of the LHC clock remains stable with respect to the bunch arrival times at the location of the $\mathrm{LHCb}$ detector

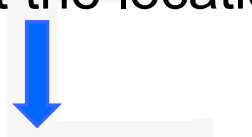

Monitoring performed using two Beam Pick-Up Timing Experiments (BPTXs) dedicated to LHCb and located along the LHC ring: formed by 4 electrostatic button electrodes producing a bipolar signal which is the representation of the beam (bunch-by-bunch) inside the beam pipe
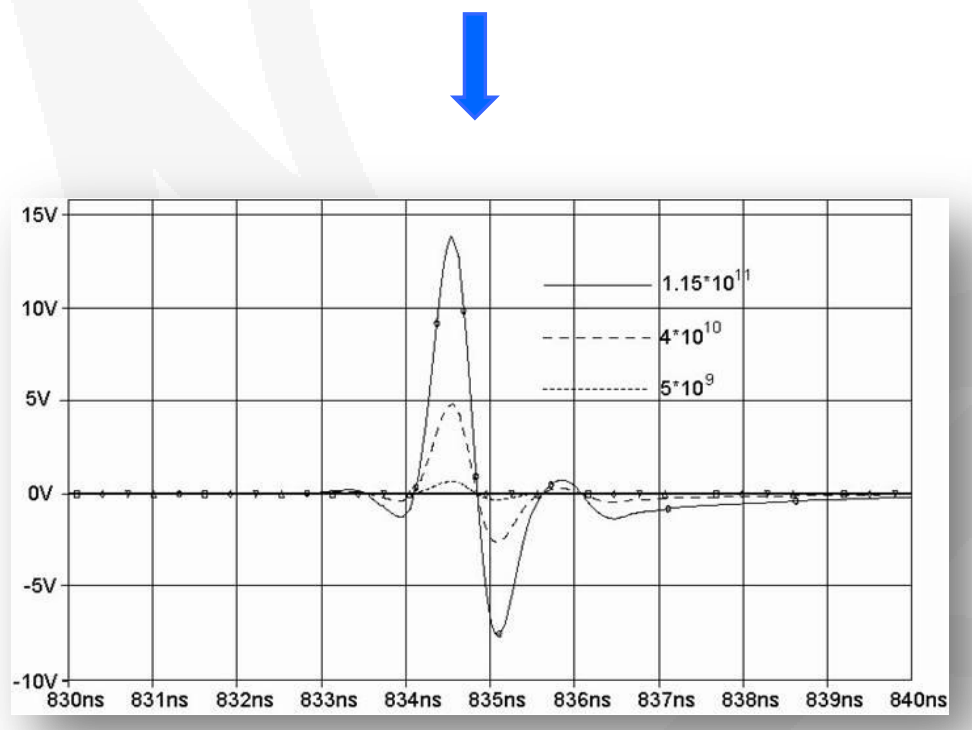

TWEPP2010, Aachen, Germany, 24/09/10
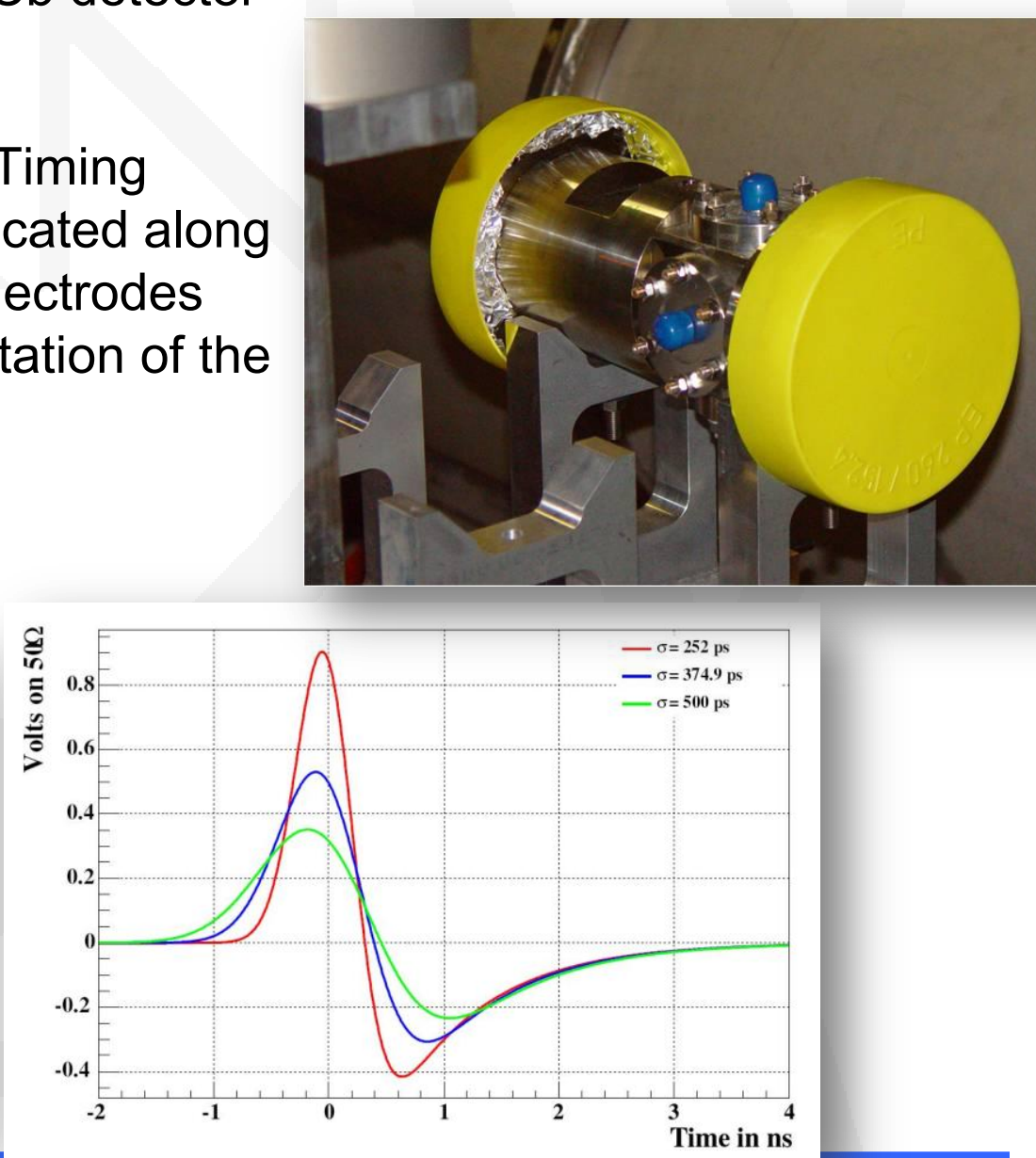

F. Alessio, CERN 


\section{LHCb BLS system}

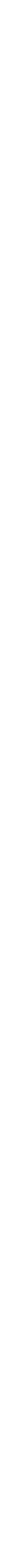




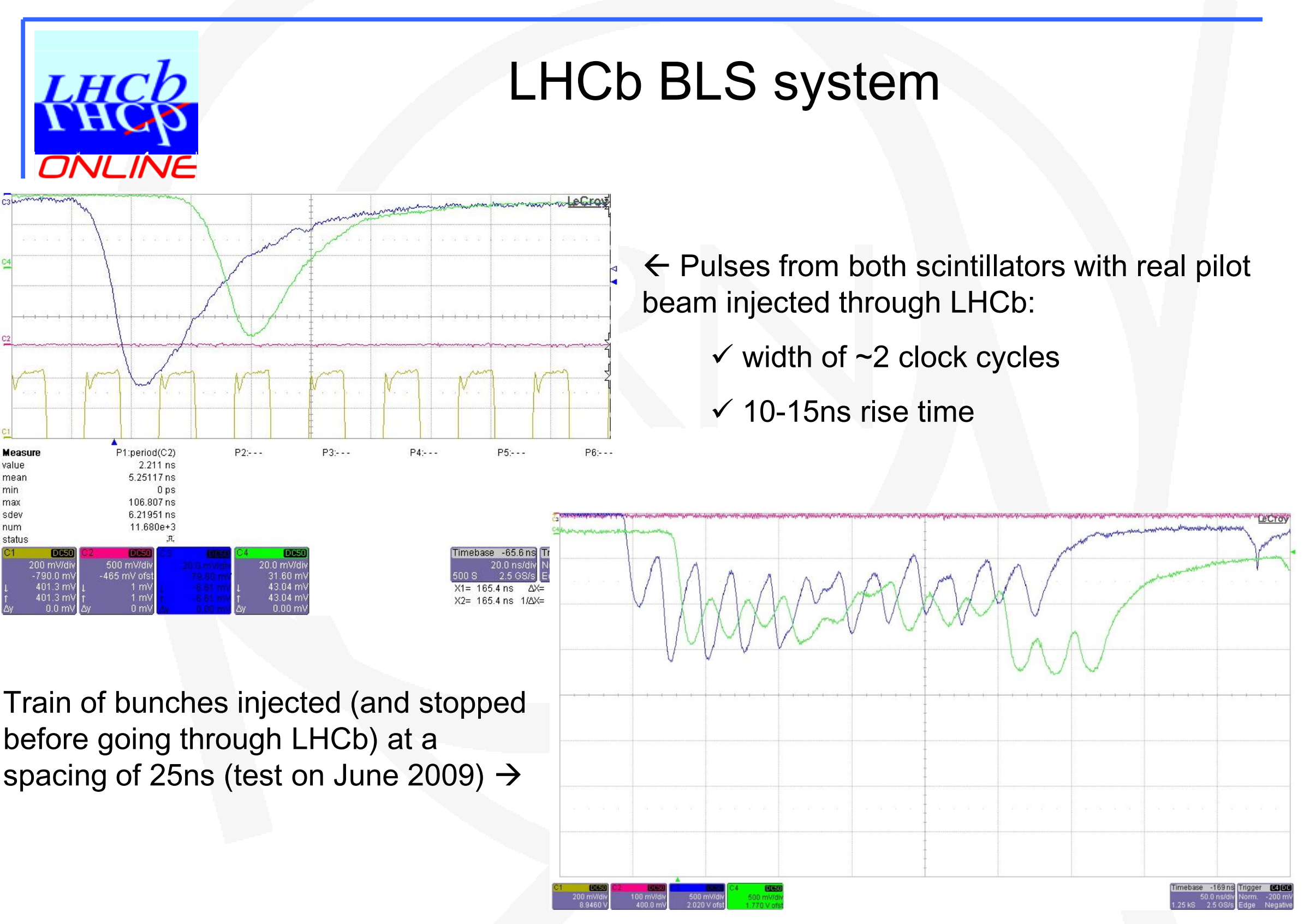




\section{Beam and Background FOM @ LHCb}

ONLINE

BPIM-BPTX

- $\quad$ Beam intensity. independent LHCb measurement of total beam intensity and bunch-by-bunch intensity to be used for offline analysis and luminosity correction

- $\quad$ Bunch structure: so-called LHC filling scheme

- Beam phase with respect to sampling clock edge: keep the LHCb detector within +/- 0.5ns from optimal sampling point

BLS.

- Beam losses:

$\checkmark$ Losses during injection: LHCb under the fire of showers at injection

$\checkmark$ Losses during circulating beams: beam Halo, fast losses due to scraping, losses due to beam movements, increasing background, beam-beam effects, etc...

$\checkmark$ Abort gap monitoring: look for ghost bunches, beam debunching due to RF, wrong filled bunches

- $\quad$ LHCb independent luminosity measurement: cross-check with official LHCb luminosities measurement (from LOCALO trigger rate) and substitute LHCb official measurement whenever not available (detector not running or not ready during collisions). Bunch by bunch luminosity. Both:

- Live feedbacks to $\angle H C b$ control room and $\angle H C$ control room for shifters: automation, alarms, archiving! electronics $\angle H C b$ board 


\section{LHCb A common electronics board: "BPIM"}

ONLINE

$\rightarrow \quad$ Initial spec: custom-made acquisition board

$\checkmark 6 U$ VME. LHC orbit and bunch clock inputs.

$\checkmark$ Online analysis of a bipolar pulse: FWHM $1 \mathrm{~ns}$ at $40 \mathrm{MHz}, \pm 5 \mathrm{Vmax}$ processing amplitude (onboard attenuator for higher pulses)

$\checkmark$ Measure time between bunch arrivals and LHC bunch clock locally

- Bunch-by-bunch for a full LHC turn filled in FIFO

- Triggered via controls interface

- $<100$ ps precision and averaging phase as a function of bunch crossing (TDC-GPX, 27ps)

$\checkmark$ Measure continuously bunch intensities bunch-by-bunch

- 12-bit resolution by integrating pulse per bunch (pipelined ADC)

- Output intensity on front-panel at $40 \mathrm{MHz}$ (8/4-bit resolution)

- Triggered via controls interface, fill in FIFO with intensities for full turn

- Intensity per bunch as a function of bunch crossing

$\checkmark$ Readout and control via Experiment Control System, CCPC based interface (VME alternatively). Glue logic for interfacing protocols.

$\checkmark$ Interfaced directly to LHCb Timing and Fast Control system, 3 outputs

$\checkmark$ Data processing on FPGA (ALTERA EP20K200, with 8000 LEs), two 16bx16b FIFOs for storage, EEPROM for firmware storage and load

$\checkmark$ Initially only developed for BPTX system 


\section{BPIM timeline}

$\rightarrow$ September 2006, first conceptual presentation by R. Jacobsson at $12^{\text {th }}$ LECC in Valencia: PSPICE simulations, first PCB draft

$\checkmark$ http://cdsweb.cern.ch/record/989402/files/lhcb-2006-055.pdf

$\rightarrow$ February 2007, first PCB produced, mounting started, lots of debugging and fixing in first prototype

$\rightarrow$ May 2007, second prototype produced and mounted, tests in lab

$\rightarrow$ September 2007, tests in SPS as readout board on a real BPTX:

$\checkmark$ http://cdsweb.cern.ch/record/1082870/files/CERN-THESIS-2008-007.pdf

$\rightarrow$ January 2008, production of a batch of 8 boards

$\rightarrow$ September 2008, BPIM sees first beam reading out first BPTX pulse

$\checkmark$ Some adaptation neede because performances of BPTX different from signal generator

$\rightarrow$ January 2009, 3 BPIMs go to ALICE (waiting for beam...)

$\rightarrow$ July 2009, 2 BPIMs installed in BLS readout (waiting for beam...)

$\rightarrow$ October 2009, all BPIMs see beam again

$\rightarrow$ May 2010, commissioning with LHCb detector, LHCb timing adjusted, BLS was first detector to signal $3.5 \mathrm{TeV}$ collisions in point 8

$\rightarrow$ Continuous monitoring and analysis of data 


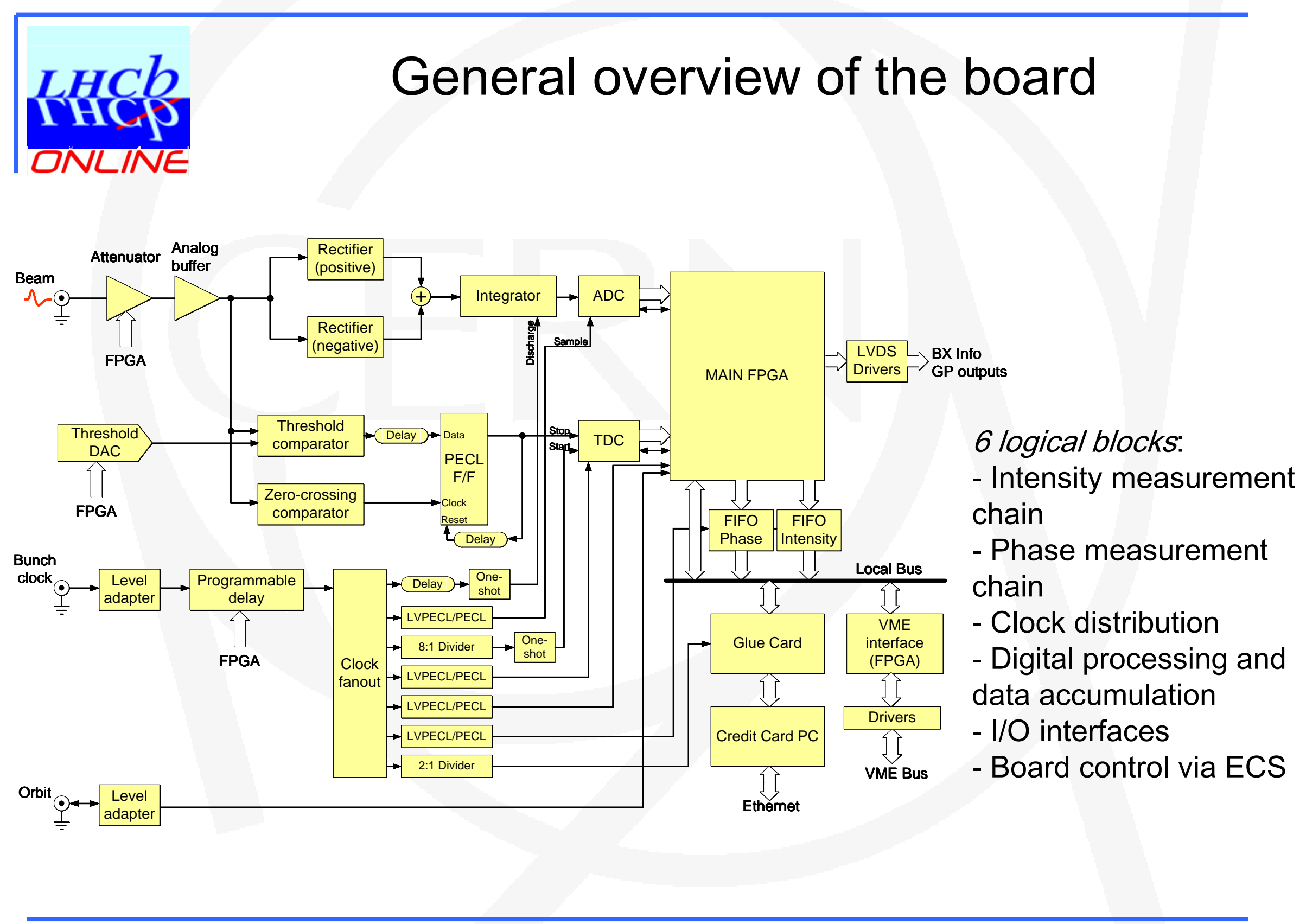




\section{In depth: analog chain}

\section{ONLINE}

Analog chain for the intensity measurement, involving current amplifiers, buffers and integrator
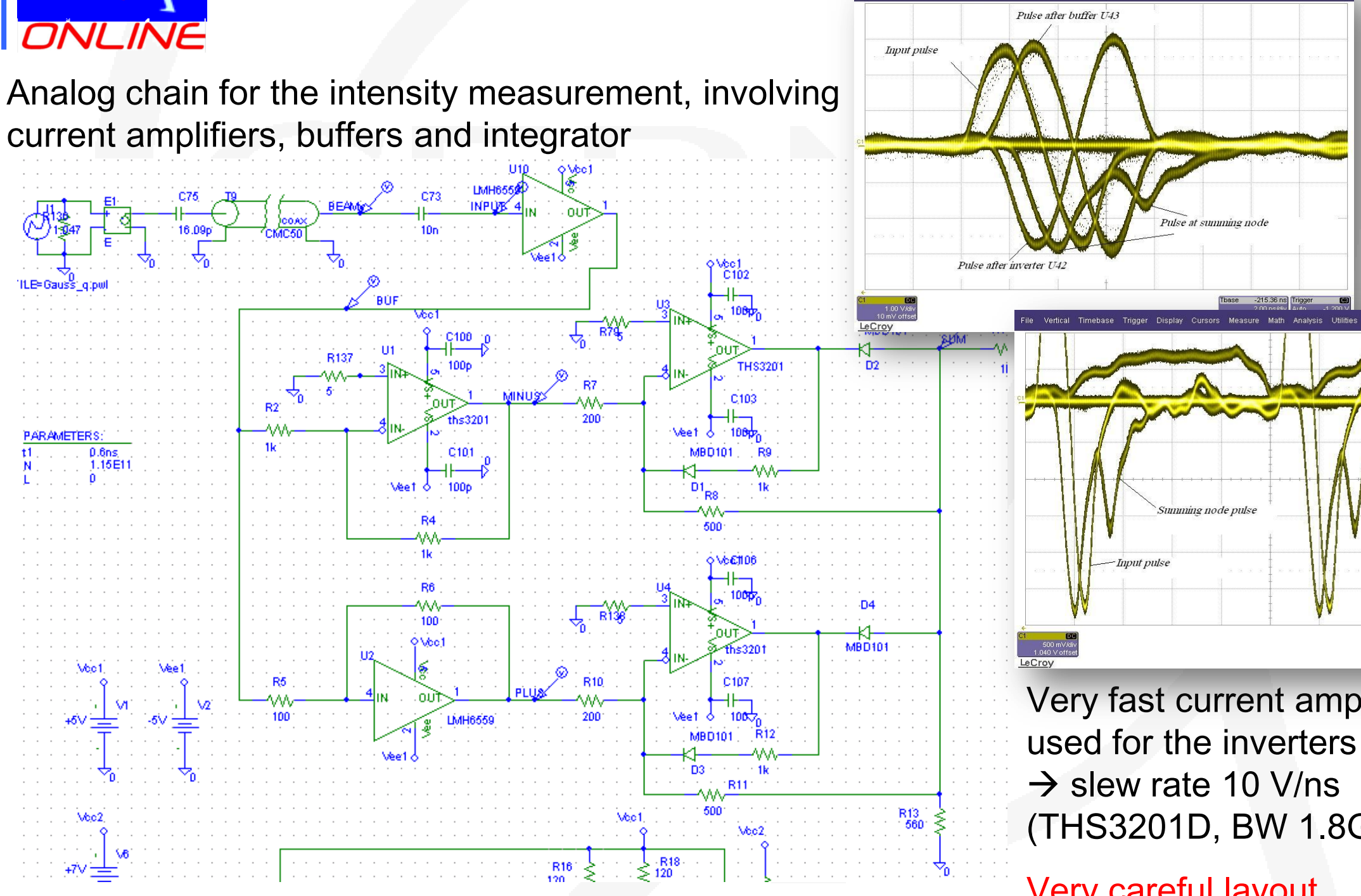

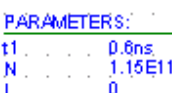
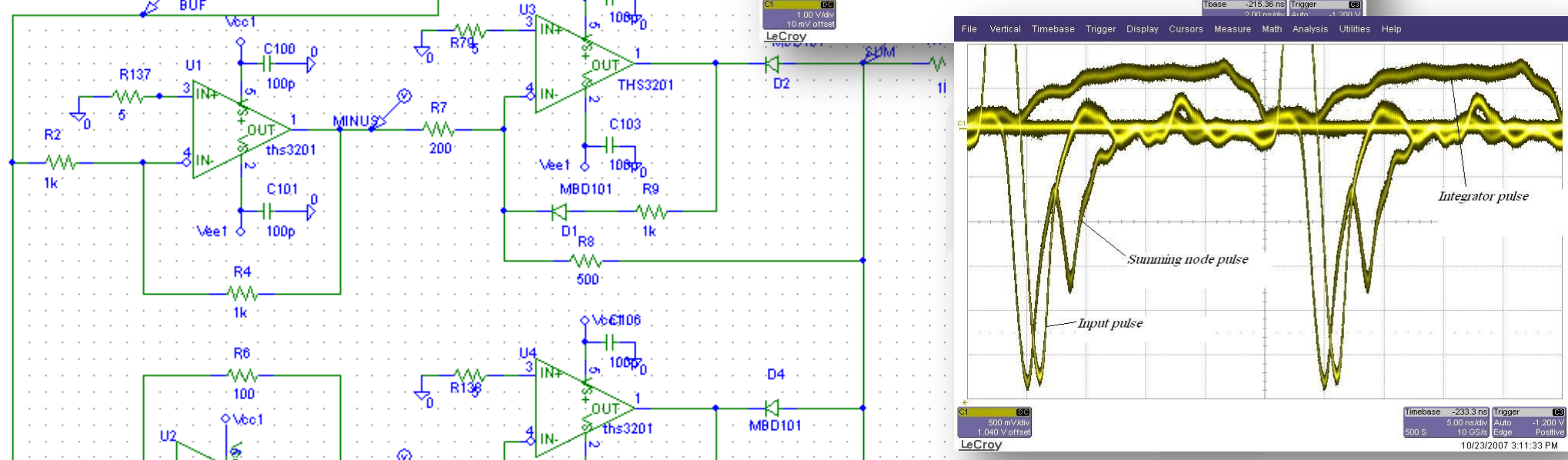

Very fast current amplifier used for the inverters stages $\rightarrow$ slew rate $10 \mathrm{~V} / \mathrm{ns}$ (THS3201D, BW 1.8GHz)

Very careful layout 


\section{Performances with real LHC beam}

LHCb

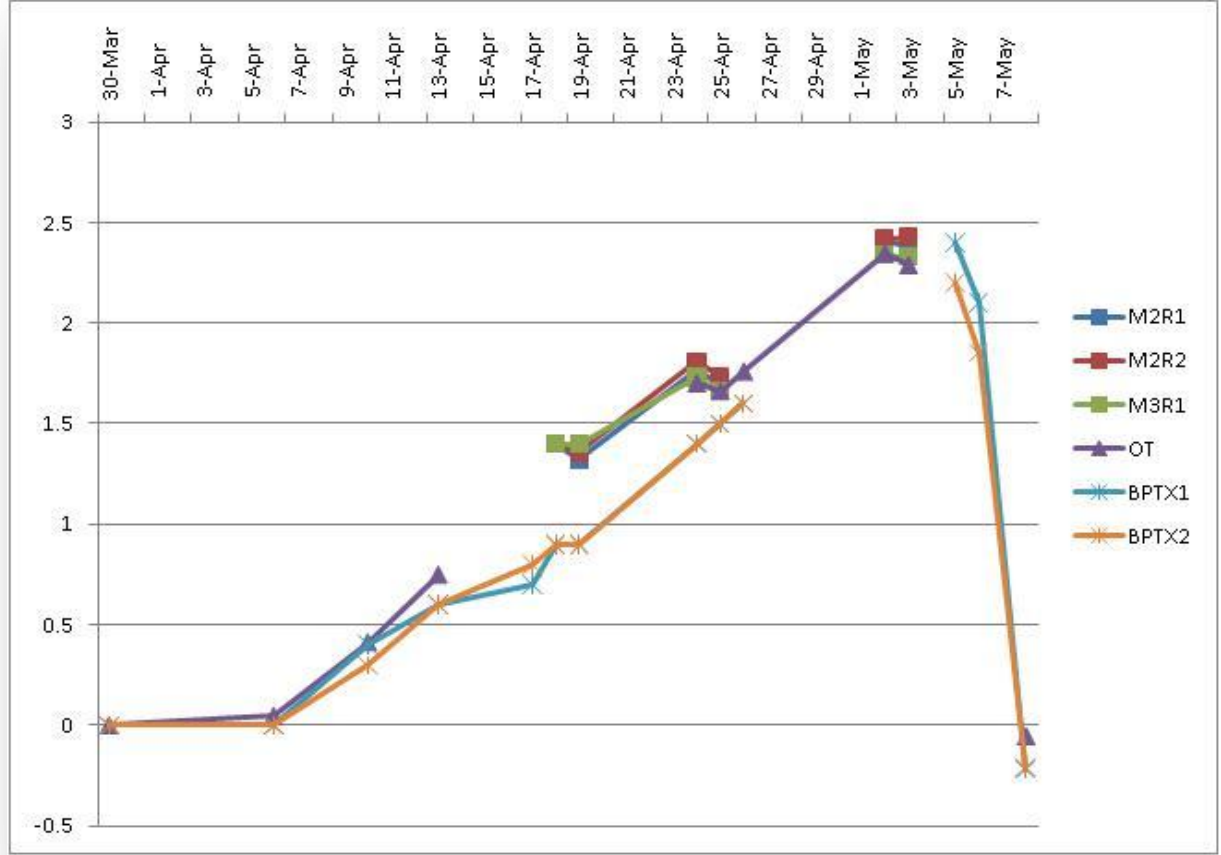

$\rightarrow$ LHCb experienced a drift of about $2.3 \mathrm{~ns}$ over a period of about 1 month (March May 2010)

$\checkmark$ Phase of the sampling clock edge drifted because of temperature drift (via fibres)

$\rightarrow$ Same drift was observed by two detectors in LHCb equipped with TDCs (Outer Tracker and MUONs stations)

$\checkmark$ Commissioning phase of BPIM and calibration with $\mathrm{LHCb}$ detectors

$\checkmark$ Realignment of the phase

$\rightarrow$ For LHCb a positive shift $(+)$ corresponds to a delay of the clock edge with respect to the beam passage

$\checkmark \quad$ the beam is earlier with respect to our optimal sampling point 


\section{LHCb Performances with real LHC beam}

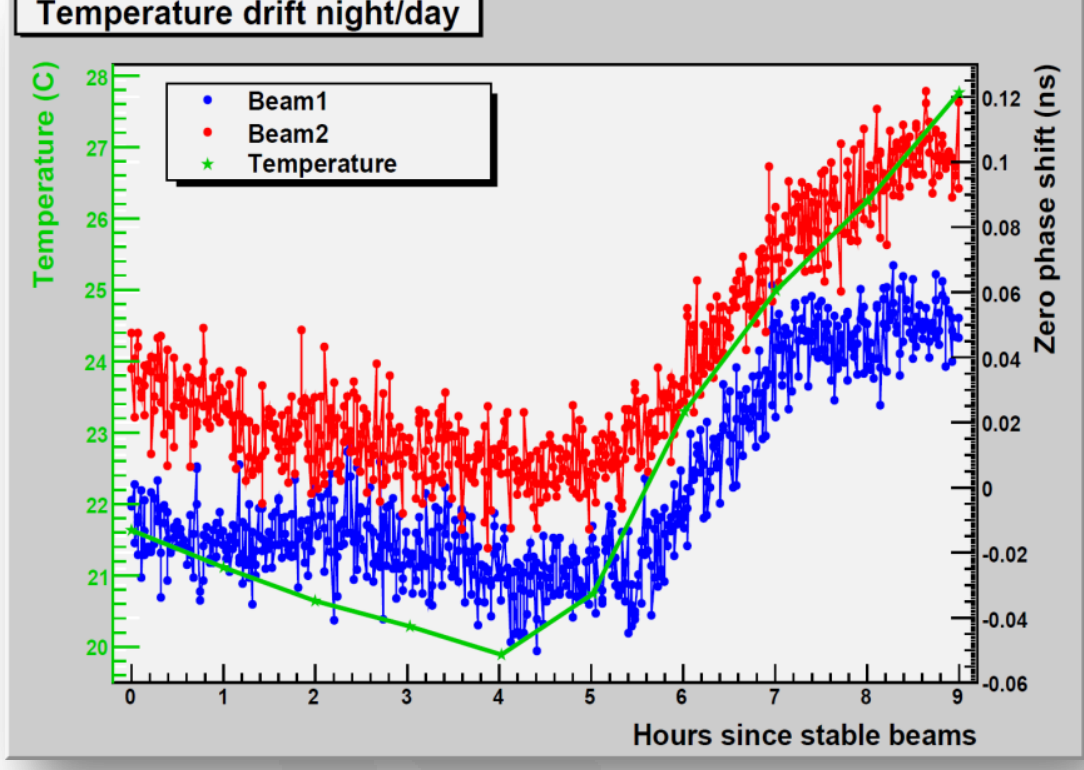

$\rightarrow \quad$ LHC Fill 1222

$\checkmark$ Temperature drifted by $8 \mathrm{C}$ in 5 hours

$\checkmark \quad$ Beam1 and beam2 clock phase shifted by $\sim 100 \mathrm{ps}$

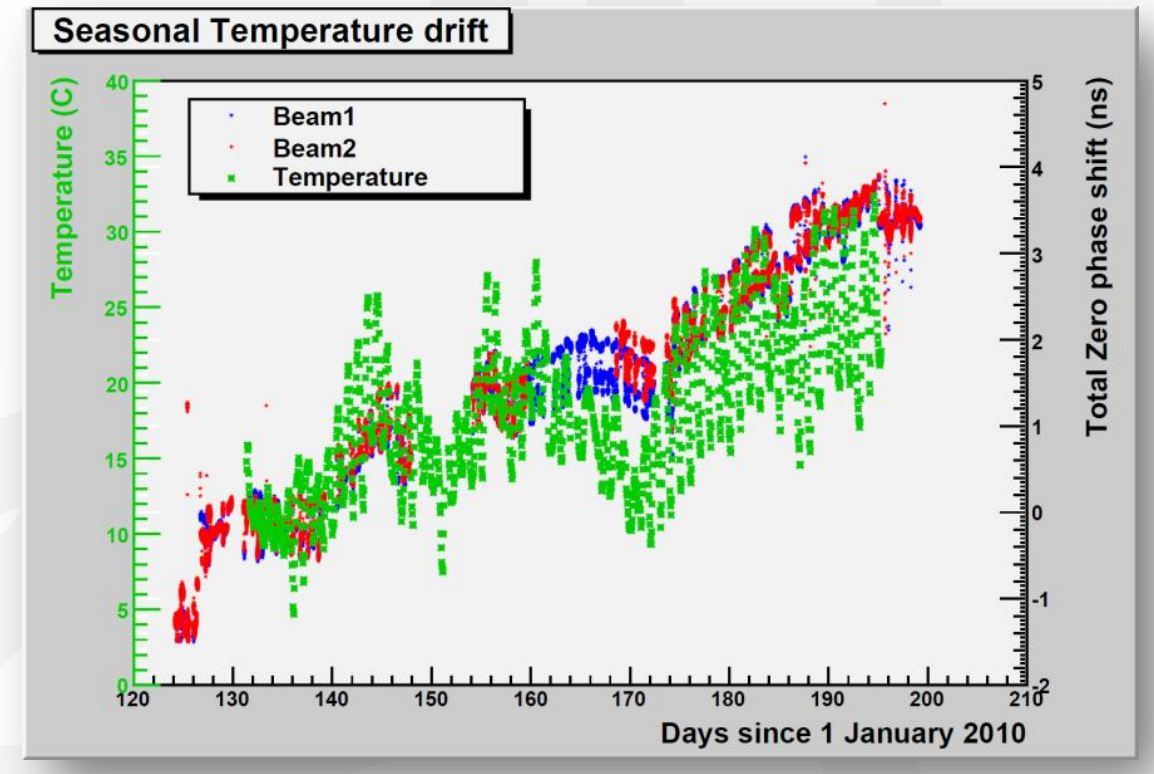

$\rightarrow 90$ days running period

$\checkmark$ Temperature drifted by $15 \mathrm{C}$

$\checkmark$ Clock edge phases drifted by $\sim 4.5 \mathrm{~ns}$

$\checkmark$ Average of $260 \mathrm{ps} / \mathrm{deg}$ over the $13 \mathrm{~km}$ of fibres from RF4 to LHCb 


\section{LHCb Performances with real LHC beam}

\section{ONLINE}

2. Resolution of phase measurement ...
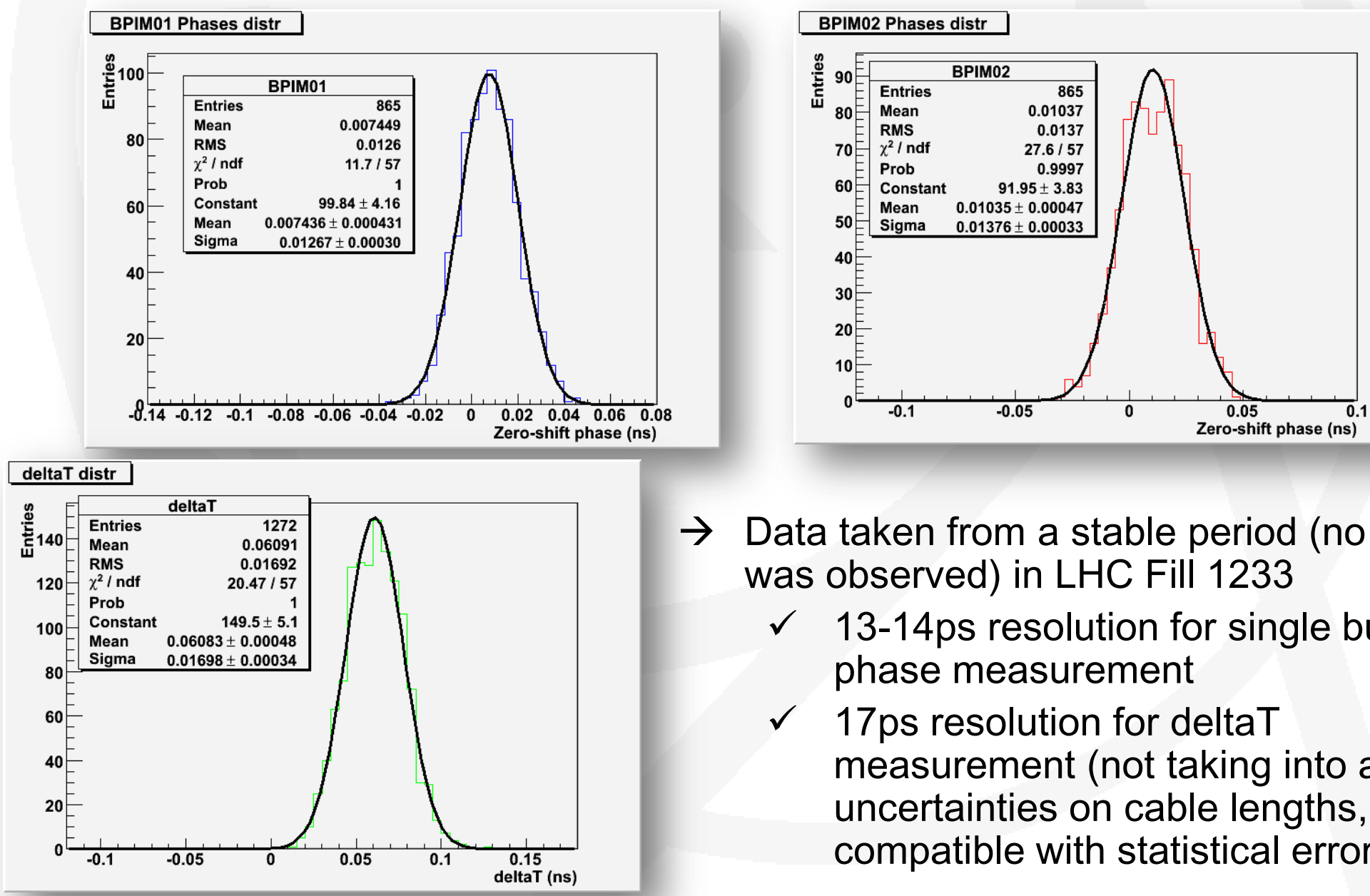

$\rightarrow$ Data taken from a stable period (no drift was observed) in LHC Fill 1233

$\checkmark \quad 13-14 p s$ resolution for single bunch phase measurement

$\checkmark \quad 17 p s$ resolution for deltaT measurement (not taking into account uncertainties on cable lengths, compatible with statistical error) 


\section{LHCb Performances with real LHC beam}

ONLINE

3. Dependance on beam intensity ...
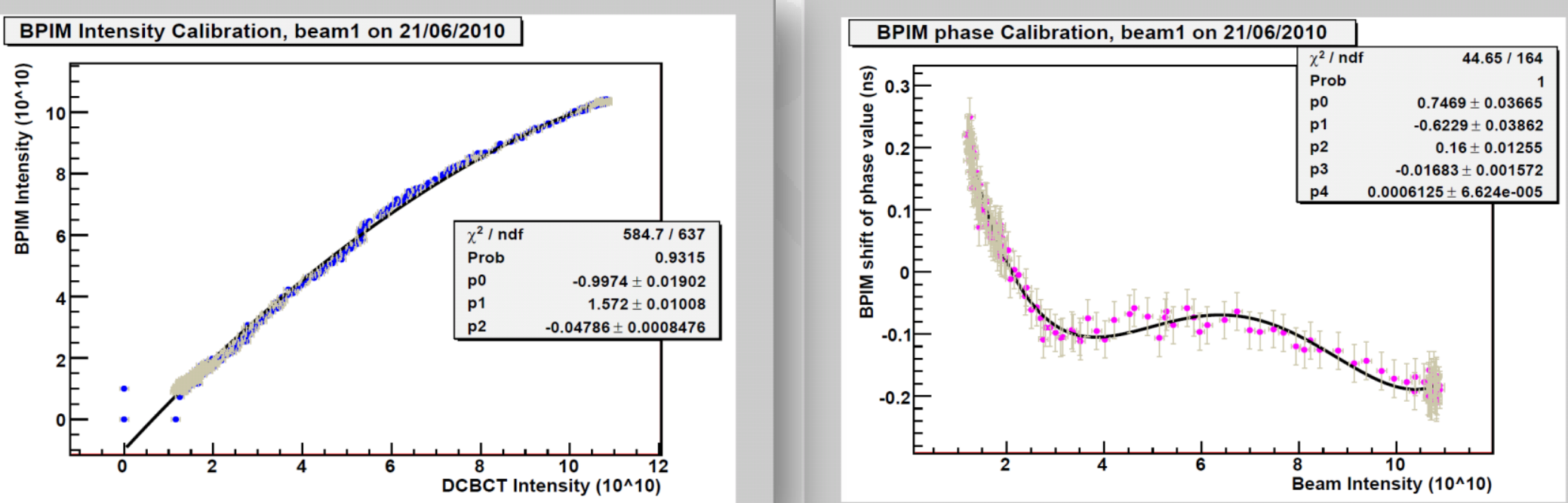

$\rightarrow$ Strong non-linearities

$\checkmark$ Correction curves, difficult to reproduce (only few cases). Need more system calibration!

Intensity resolution: (data taken from LHC Fill 1250)

$\rightarrow$ For BPIM01: $1 \mathrm{ADC}=1.4345^{*} 10^{\wedge} 8 \mathrm{ppb}$

$\rightarrow$ For BPIM02: $1 \mathrm{ADC}=1.7391 * 10^{\wedge} 8 \mathrm{ppb}$

$\checkmark$ Due to baseline subtraction ( $\sim 20 \mathrm{ADC}$ counts above baseline), the minimum intensity measurable by the system is $\sim 4^{*} 10^{\wedge} 9 \mathrm{ppb}$.

$\checkmark$ Reflections in the cables when high intensity beam: raise threshold above baseline 


\section{LHCb Performances with real LHC beam}

ONLINE

4. Commissioning of the injection transfer lines and TDI settings ...
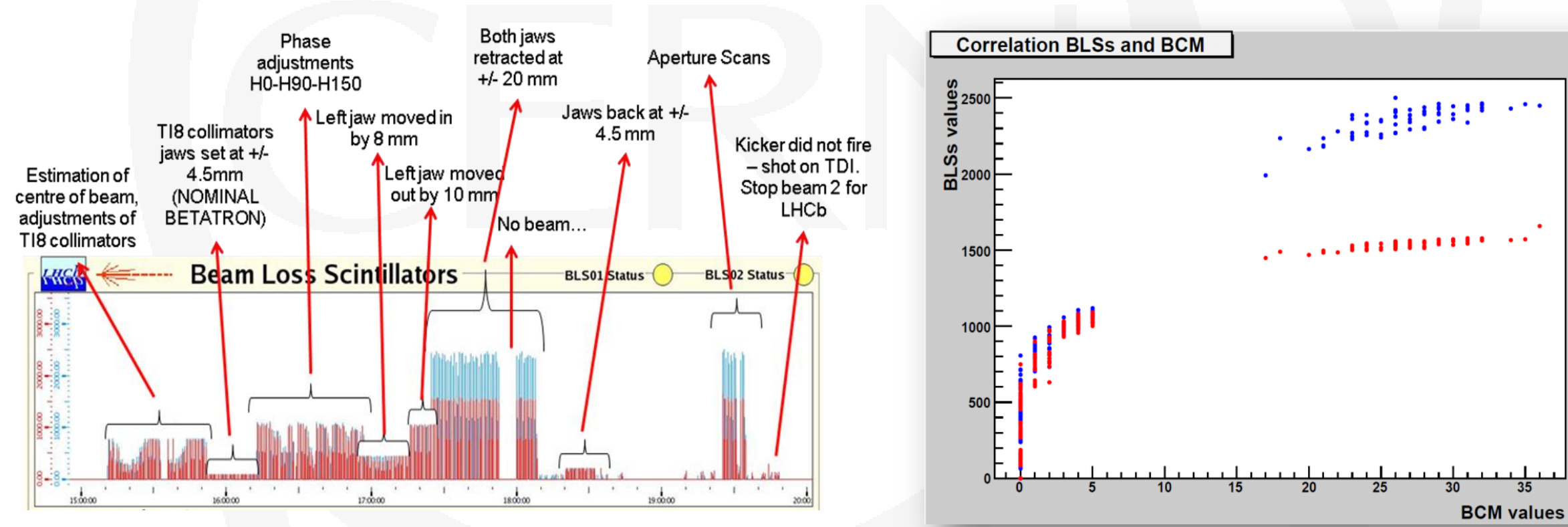

$\rightarrow$ Possible to "calibrate" the position of the collimators with "desired" losses

$\rightarrow$ Whole range of losses is covered by BLS and BCM

$\checkmark$ BLS more sensitive than BCM

$\checkmark$ BLS saturates at $10 \%$ of threshold BCM 


\section{LHCh}

Performances with $\mathrm{r} \epsilon$

ONLINE

\section{Luminosity measurements...}

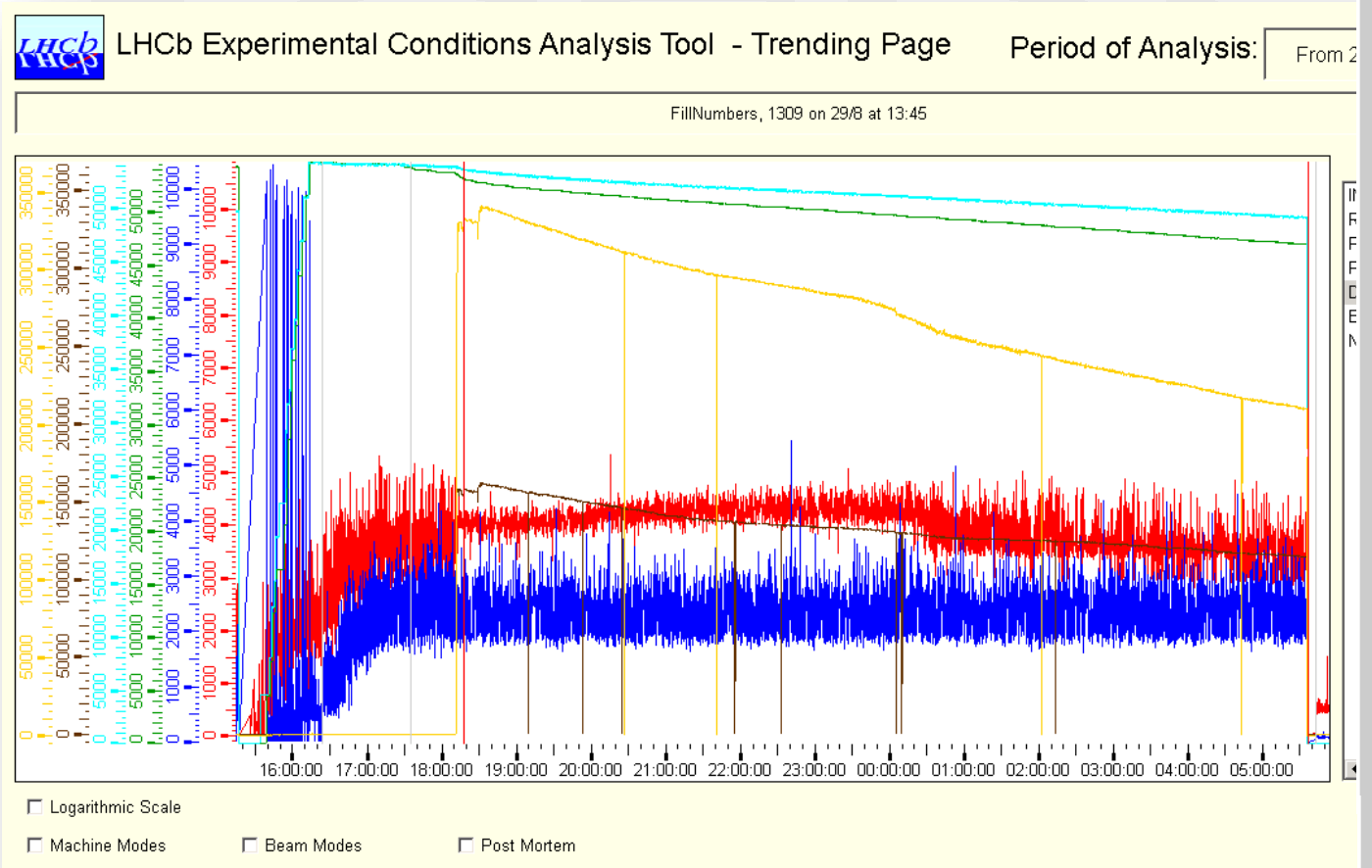

$\rightarrow$ Different behaviour according to different machine modes

$\checkmark$ losses to be studied systematically and accurately

$\rightarrow$ Need more calibration: scintillators are already ageing because non rad-hard, source of background are well described by simulation and in agreement, translate ADC in \# of MIPs.

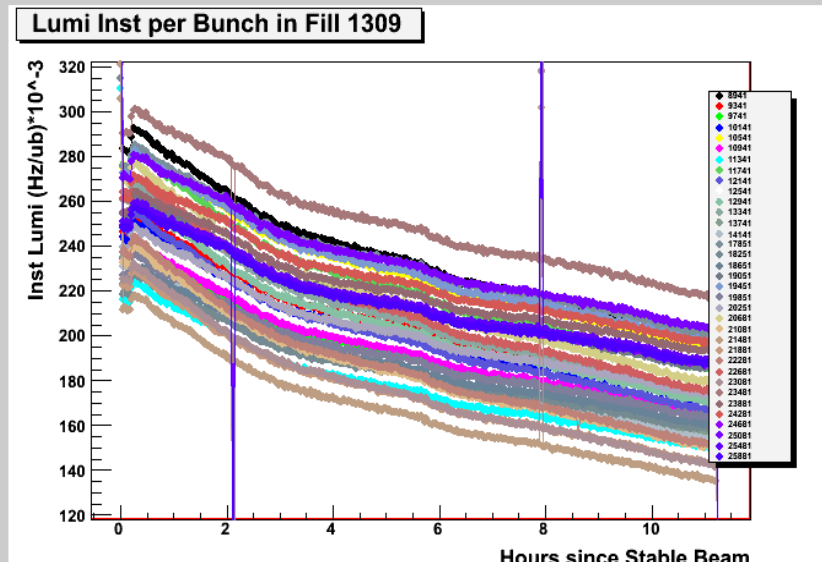

Hours since Stable Beam
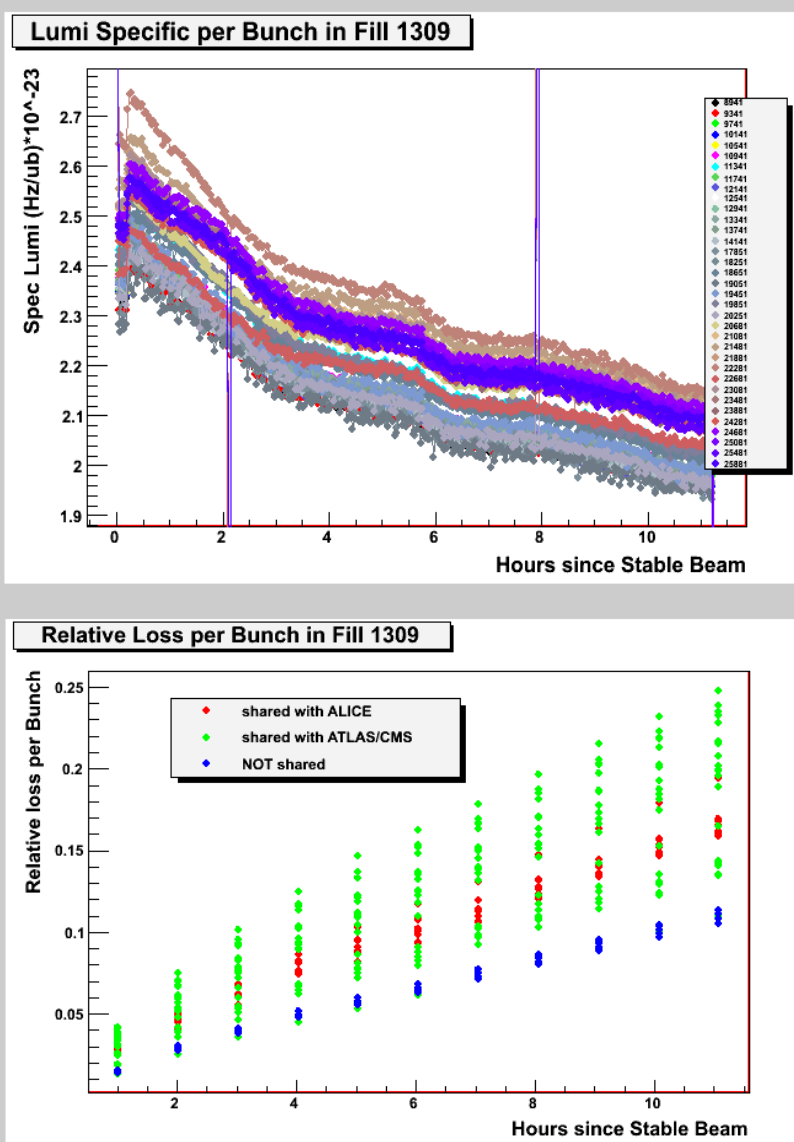


\section{BPIM in ALICE}

$\rightarrow$ "Bunch masking"

$\checkmark$ Coincidence of two BPIMs (per beam) == collisions

$\checkmark$ Part of the "ALICE SMAQ" plot (OBPA and OBPB): quickest trigger in ALICE!

$\rightarrow$ Modification in order to see also pilot bunches

$\checkmark$ Amplification modified by factor $\sim 4$

$\rightarrow$ Efficiency 100\%: never missed a trigger!

$\rightarrow$ Ready for lon runs in ALICE: extremely important to have the system ready since day 1

$\rightarrow$ The boards have proven to be working in a radiation environment without being affected by it

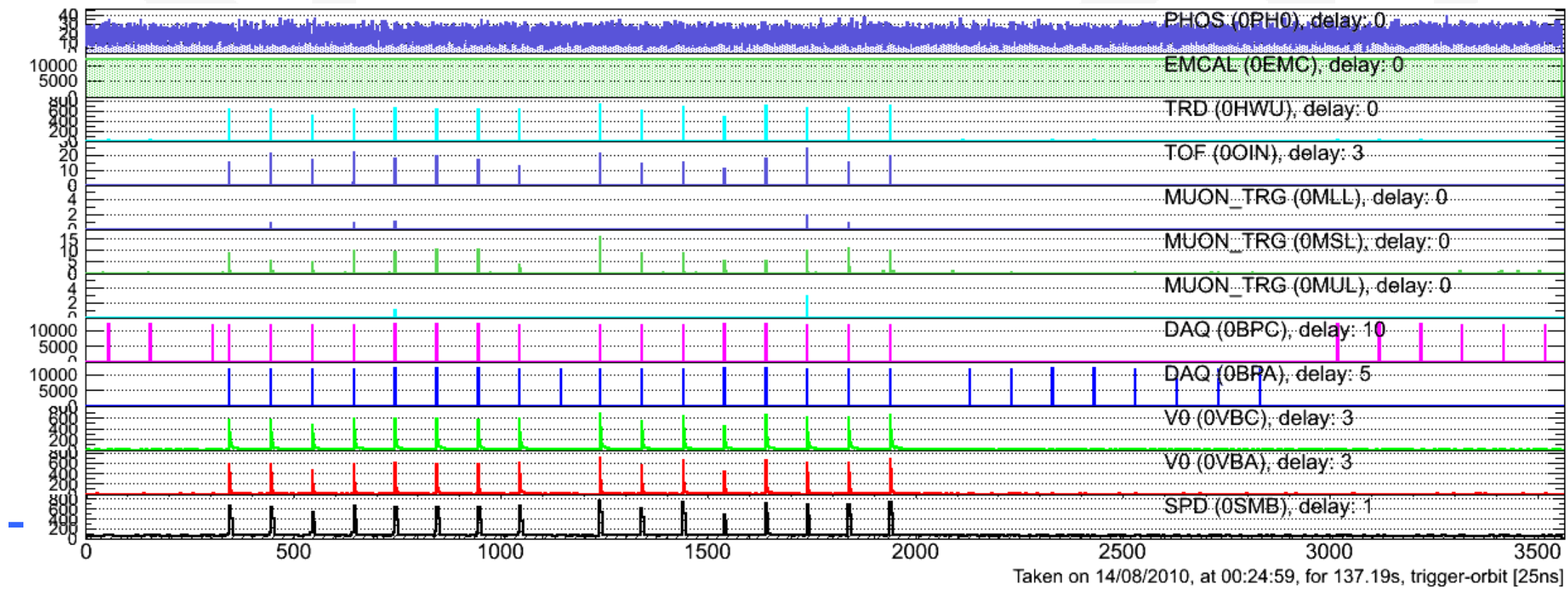




\section{$\mathrm{LHCb}$ Control, monitoring and data presentation \\ rнp}

ONLINE

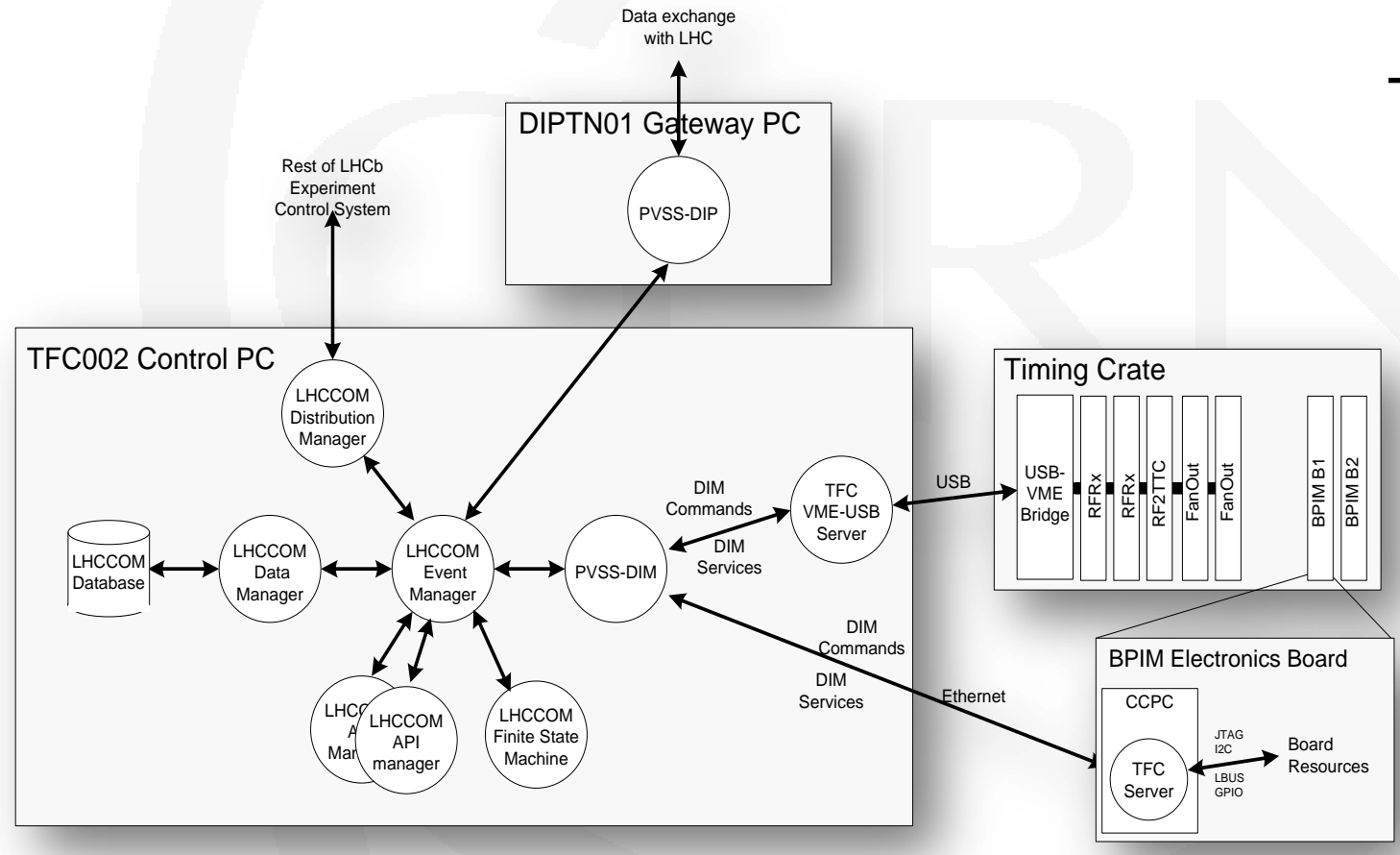

$\rightarrow$ System allow for generic subscription to monitoring information for continuous archiving and permanent display in the $\mathrm{LHCb}$ control room $\rightarrow$ Based on Credit Card-sized PC with Ethernet running a stripdown version of Linux

$\checkmark$ Access board resource via PCl bus converted to native hardware busses by glue logic

$\checkmark$ Server based on the same generic protocol on top of DIM allows the PVSSbased control system to configure and monitor the electronics 


\section{$\mathrm{LHCb}$ Control, monitoring and data presentation}

ONLINE
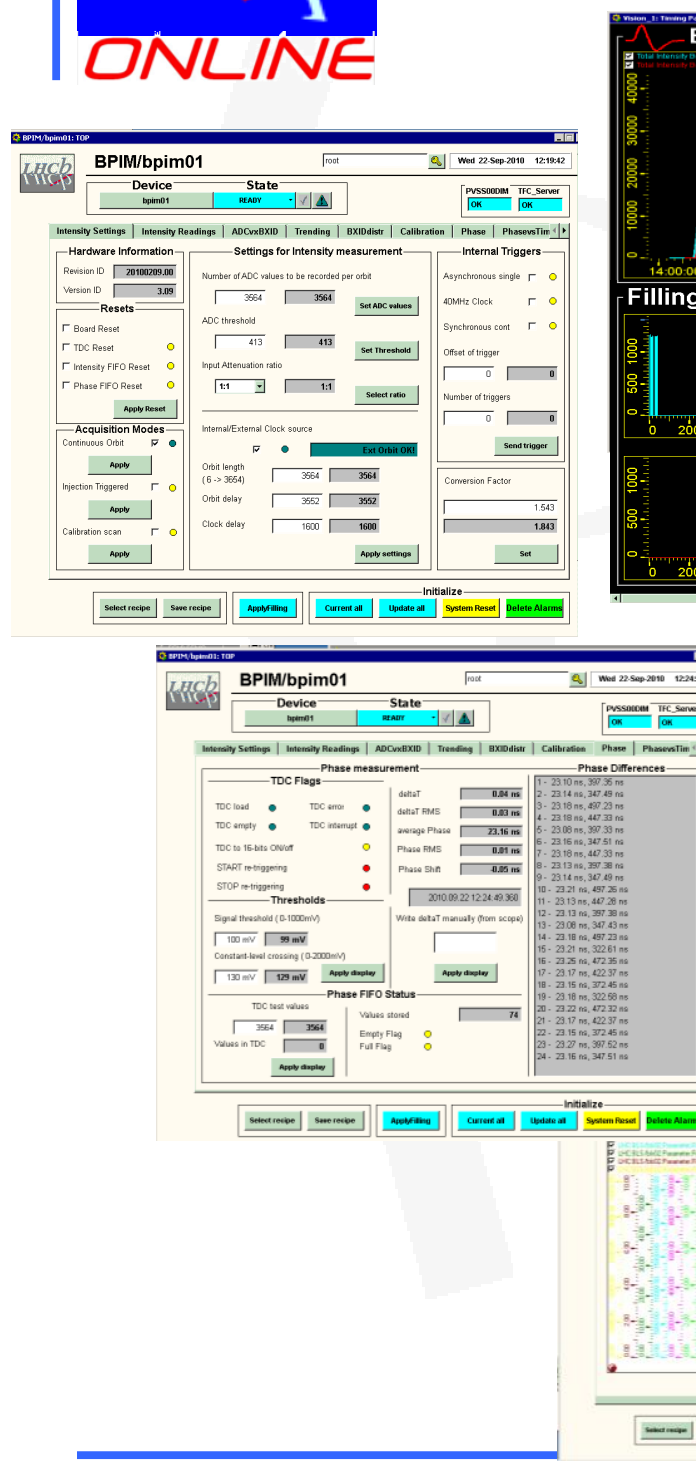

TWEPP2010, Aachen, Germany, 24/09/10
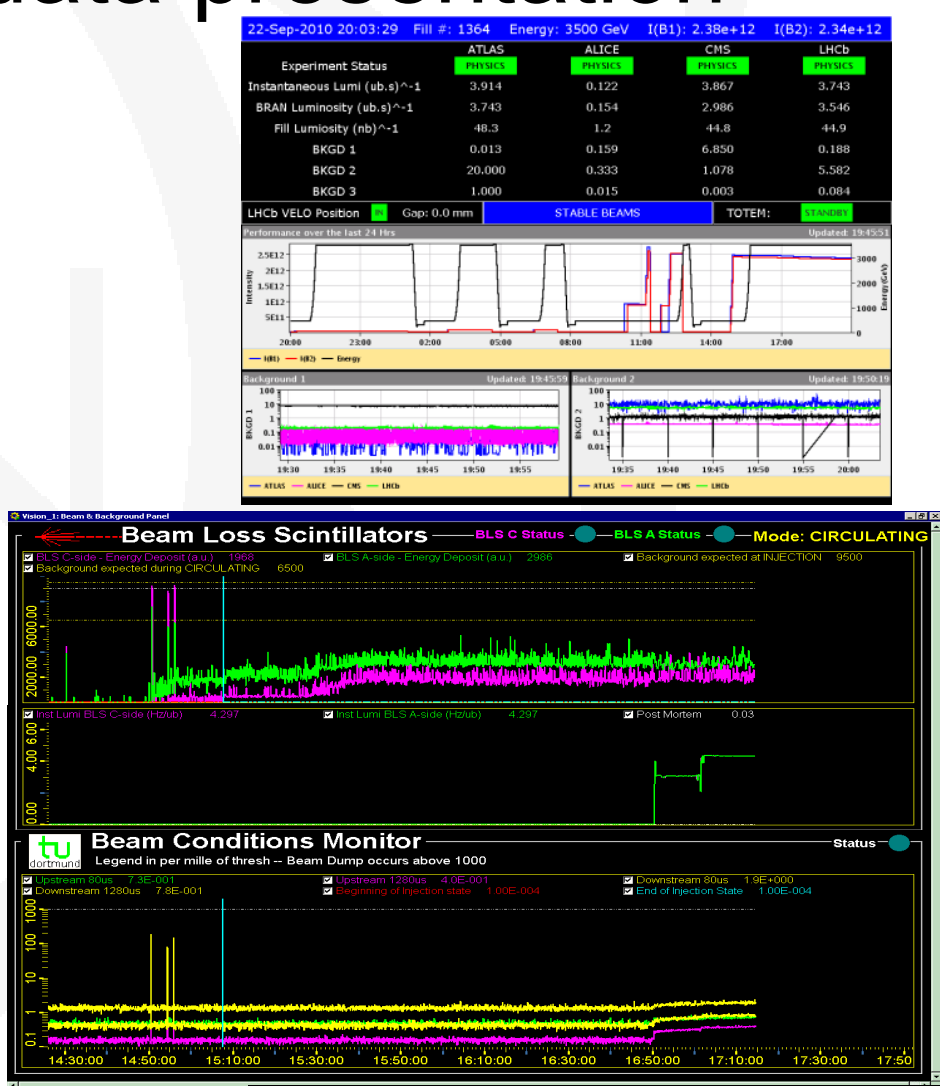

LHCb Luminosity Rates / Instantaneous / Ratio to Theoretical

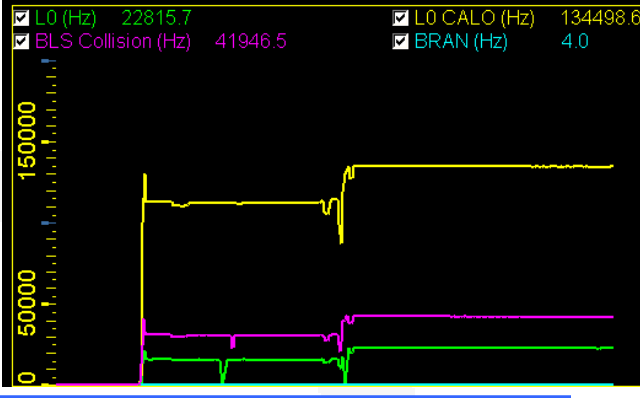




\section{Conclusions}

$\rightarrow$ LHCb has developed, produced and put in operation an extremely flexible, reliable and automated multi-purpose high performance acquisition board

$\rightarrow$ Proven its great performance with first real LHC beam

$\checkmark \quad$ First resource in $\mathrm{LHCb}$ for monitoring timing

$\checkmark \quad$ First resource in LHCb for monitoring LHC filling scheme and possible ghost bunches

$\checkmark$ First resource in LHCb for bunch-by-bunch dedicated measurements (intensity, phase, instantaneous and specific luminosity per bunch)

$\checkmark$ Readout card for a LHCb scintillators system dedicated to beam and background monitoring

$\checkmark$ Part of the ALICE trigger chain

$\rightarrow$ Future plans:

$\checkmark$ Make a second production batch

$\checkmark$ Expand BLS with more scintillators (rad-hard)

$\checkmark$ Finally put a front panel... 